\title{
Etat Oceanographique De La Lagune De Oualidia, Maroc (2011-2012)
}

\author{
Ahmed Makaoui, \\ Mohammed Idrissi, \\ Aziz Agouzouk, \\ Jamila Larissi, \\ Tarik Baibai, \\ Zineb El Ouehabi, \\ Département d'Océanographie, \\ Institut National de Recherche Halieutique (INRH), Casablanca, Maroc \\ Mohamed Ait Laamel, \\ Direction de Météorologie Nationale (DMN), Casablanca, Maroc. \\ Ismail Bessa, \\ Département de physique, Faculté des Sciences Ben M'Sik, \\ Université Hassan II Casablanca, Maroc

\section{Omar Ettahiri, Karim Hilmi,} \\ Département d'Océanographie, \\ Institut National de Recherche Halieutique (INRH), Casablanca, Maroc
}

Doi: 10.19044/esj.2018.v14n18p93 URL:http://dx.doi.org/10.19044/esj.2018.v14n18p93

\begin{abstract}
The study of the Oualidia lagoon aims to establish the state of knowledge of the environment of this ecosystem after the construction of an inner dam in 2011 and the ecological functioning of the lagoon. For that, several campaigns were carried out between February 2011 and October 2012 and were the subject of a seasonal monitoring for the collection of the main biotic and abiotic parameters of the environment of this ecosystem. During the construction of the inner dam on 2011, a hydrological variability was marked in summer by low oxygen levels and maximum temperature variation between the two tides. Since the completion of the construction of the inner dam, there has been a significant increase in nutrients, mainly nitrogenous elements, and an increase in turbidity caused by an improvement in hydrodynamics, which has particularly increased of the sandpit modified the morphology and moved on south, creating a new main channel structure in 2011. This hydrodynamic was manifested on the upstream area by a decrease of the sedimentary organic
\end{abstract}


matter due to the trapping of fine sediments in the inner dam. At the immediate level of the upstream area (Slikkes and Schorres), the hydrodynamic is relatively low, which has reduced the sedimentation rate on the upstream of the lagoon. The Oualidia lagoon is situated in a littoral zone influenced by the enrichment in deep cold waters loaded with nutritive salts, which invade it during the high tide, and would be, consequently after construction of the inner dam, protected from any confinement of the ecosystem.

Keywords: Oualidia lagoon, Hydrodynamic, hydro sedimentary variability, lagoon waters

\section{Résumé}

Cette étude menée sur la lagune de Oualidia a pour objectif de dresser l'état des connaissances du milieu de cet écosystème après la construction d'une souille en 2011 ainsi que le fonctionnement écologique de la lagune. A cet effet, Plusieurs campagnes ont été effectuées entre février 2011 et octobre 2012 et ont fait l'objet d'un suivi saisonnier pour la collecte des principaux paramètres biotiques et abiotiques du milieu de ce site. Pendant la période de la construction de la souille en 2011, la variabilité hydrologique a été marquée en été par de faibles teneurs en oxygène et une variation maximale de la température entre les deux marées. Depuis l'achèvement des travaux de construction de la souille, il a été constaté, une élévation importante des nutriments, principalement en éléments azotés, et une augmentation de la turbidité causée par une amélioration de l'hydrodynamisme qui s'est accentué notamment au niveau de la sablière qui a changé de morphologie et qui s'est déplacée vers le sud en créant une nouvelle structure du chenal principal en 2011. Cet hydrodynamisme s'est manifesté en amont par une diminution de la matière organique sédimentaire qui est due au piégeage des sédiments fins dans la souille. Au niveau immédiat de la zone amont des slikkes et des schorres, l'hydrodynamique assez faible a subi un changement, ce qui a diminué le taux de sédimentation en amont de la lagune. La lagune de Oualidia est située dans une zone littorale influencé par l'enrichissement en eaux froides profondes chargées en sels nutritifs qui l'envahissent pendant la marée haute, et serait, par conséquent après construction de la souille, à l'abri de tout confinement de l'écosystème.

Mots clés: Lagune de Oualidia, Hydrodynamique, variabilité hydro sédimentaire, eaux lagunaires

\section{Introduction}

La lagune de Oualidia est l'un des sites au Maroc où l'ostréiculture s'est développée de manière artisanale depuis les années 1950. Elle s'inscrit 
dans un domaine de $8 \mathrm{~km}$ (avec la souille) de long sur 0,4 à $0,5 \mathrm{~km}$ de large et sa superficie totale est évaluée à $3 \mathrm{~km}^{2}$ (Beaubrun, 1976). Depuis quelques années, les recherches réalisées sur ce site ont montré que la qualité des eaux commence à se détériorer suite à un confinement des eaux en amont de la lagune. Cette lagune a fait l'objet de nombreux travaux portant sur les aspects hydro-biologiques (Beaubrun, 1976 ; Orbi et al., 1998 ; Rharbi, 2000 ; Rharbi et al., 2001), sédimentologiques (Carruesco, 1989; Sarf, 1999), courantologique (Hilmi, 2005 ; Hilmi et al., 2005a et b) ainsi que sur la qualité et la salubrité du milieu marin (Chafik et al., 1996 ; El Attar 1998 ; Bennouna 1999 ; Benbrahim et al., 2015 ; Bouhallaoui et al., 2017).

La construction d'une souille, entre les deux digues, comme piège à sédiment serait un facteur qui pourrait donner à la lagune une nouvelle reprise à son état normal de salubrité des années 50. Cette étude a pour obj ectif de décrire l'état saisonnier Eco-systémique après l'arrêt de l'activité de la souille dans le but de suivre l'impact de cet aménagement sur l'hydrologie et l'hydrodynamique marine de la lagune.

Le suivi de la variabilité des paramètres physiques (température, salinité, $\mathrm{O} 2$ dissous, MES) et hydrodynamiques (sédiment et courant) vont nous permettre de caractériser les différentes zones de ce milieu paralique selon le réseau adopté en 2011 en ajoutant une station au niveau de la souille en 2012 (Fig. 1). Les résultats de cette étude vont être comparés à l'état de l'écosystème de la lagune de Oualidia pendant l'année 2011/2012 et avant la construction de la souille (Hilmi et al., 2017), notamment l'étude océanographique réalisée en mois de juin 2010.

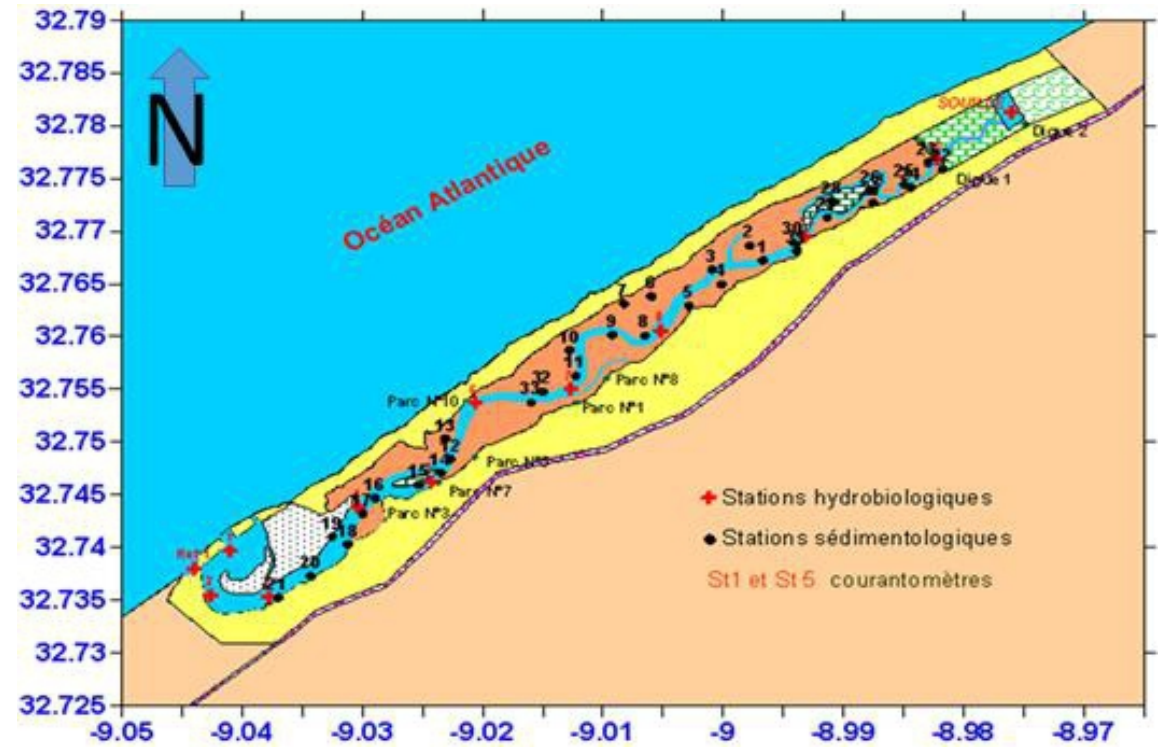

Figure 1 : Réseau d'échantillonnage des stations océanographiques dans la lagune de Oualidia réalisé sur la période 2011-2012. 


\section{Matériels et méthodes}

La prospection océanographique a porté sur l'étude physico-chimique des eaux marines à travers la collecte des paramètres physico-chimiques (température, salinité) à l'aide d'une sonde de type WTW. L'échantillonnage de l'eau s'effectue par une bouteille à renversement. L'oxygène dissous est dosé par la méthode de winkler. La matière en suspension (MES) est déterminée par filtration et la double pesée. L'analyse des nutriments est faite par un auto-analyseur AA3. Toutes les analyses ont été effectuées en laboratoire au niveau de 11 stations réparties sur l'ensemble de la lagune (S1 à $\mathrm{S} 10$ et les stations Réf et la souille) en couvrant les deux marées (MB et MH) (Fig. 1). Un courantomètre a été installé au niveau de la passe (Station S1) pendant 15 jours en couvrant une marée de vive eau et de morte eau. La détermination des faciès de la lagune et de la matière organique a été effectuée au laboratoire sur les sédiments bruts. La séparation de la fraction sableuse de la fraction lutitique a été réalisée à l'aide d'un tamis à mailles de $40 \mu \mathrm{m}$. Pour le tamisage, on a utilisé une série de 18 tamis «AFNOR » de gamme comprise entre $40 \mu \mathrm{m}$ et $2 \mathrm{~mm}$. La méthode de calcination « la perte au feu », est utilisée pour l'estimation de la teneur en matière organique présent dans le sédiment. La richesse chlorophyllienne a été déterminée par fluorimétrie.

\section{Résultats et discussion}

Le fonctionnement physique d'un milieu littoral, est choisi en fonction de l'échelle temporelle. Dans cette étude nous avons opté pour une échelle saisonnière car le fonctionnement est abordé à travers l'équilibre entre le bilan hydrique à partir des apports continentaux et des régimes des précipitation et d'évaporation, dominant en milieu lagunaire, ainsi que les échanges marins qui ont aussi un impact important sur la lagune en plus de l'impact continental (Mejjad et al., 2016). Vu les différents facteurs hydrologiques et climatologiques qui influencent l'écosystème marin de la lagune, son régime hydrologique est aussi bien climato- dépendant qu'hydro - dépendant.

Une excellente synthèse sur les vagues/houles et les vents au large de Oualidia et à proximité de la lagune a été réalisée par Koutistonsly et al. (2006). Selon ces derniers auteurs, les vents et la pression atmosphérique sont deux facteurs qui peuvent faire varier les niveaux d'eau et les courants aux passes d'entrée de la lagune de Oualidia. Les vents sont aussi responsables de la génération des vagues au large des côtes. Ces vagues se propagent vers la côte sous forme de vagues de tempête ou, en l'absence de vent, sous forme de houle (Koutistonsly et al., 2006). Les statistiques des vents du large annuels et saisonniers au large de Oualidia indiquent que i) les vents annuels moyens ont des vitesses variant entre 4 et 9 m.s-1, avec des directions prédominantes du Nord-Est et du Nord ; ii) les plus forts vents sont observés durant les mois de décembre à février, atteignant des vitesses au-delà de 30 m.s-1, surtout en 
provenance de l'Ouest et du Nord-Ouest et iii) les plus faibles vents se manifestent durant l'été et soufflent surtout du Nord et du Nord-Est (Koutitonsky et al., 2006). Pour les vents côtiers, il n'existe pas de longues séries temporelles de vent sur la lagune de Oualidia et la plupart des études font référence aux travaux de Carruesco (1989). Les directions prédominantes des vents de Mars à Août sont celles du Nord-Est et du Nord. Durant les autres mois de l'année, les vents proviennent également de ces directions mais avec des occurrences plus fréquentes du Sud-Ouest, surtout durant l'hiver (décembre à février) (Carruesco, 1989 ; Koutistonsly et al., 2006).

Toutefois, les années 2011 et 2012 restent des années à pluviométrie normale à très abondante et accompagnée par un décalage dans le temps notamment à Safi comme le montre la figure ci-dessous (Fig. 2). En effet, la pluviométrie enregistrée à Safi situé au Sud à $60 \mathrm{Km}$ était plus abondante en 2011 pendant le premier semestre, alors qu'en 2012, elle était moins abondante.

Cette situation se répercute directement sur l'activité des remontées d'eau profonde au niveau de la zone littorale de Oualidia. En effet, la côte atlantique marocaine est en action estivale du phénomène d'upwelling qui est caractérisé par des remontées d'eau profondes froides, moins salines et riches en nutriments (Makaoui et al., 2005 ; Benazzouz et al., 2014) et qui ont un impact direct sur l'écosystème des milieux paraliques notamment ceux de la lagune de Khnifiss (Lakhdar et al., 2004) et de la baie de Cintra au niveau de la zone sud (Makaoui et al., 2017) soumise à un upwelling permanent. Cette activité apparait à partir du printemps et s'intensifie en été et persiste en automne (Makaoui et al., 2012). L'indice d'upwelling dérivé de la SST et du vent (Fig. 3) montre que l'activité d'upwelling estival est plus importante en 2012 qu'en 2011 et par suite les eaux littorales sont plus froides et moins salée en 2012.

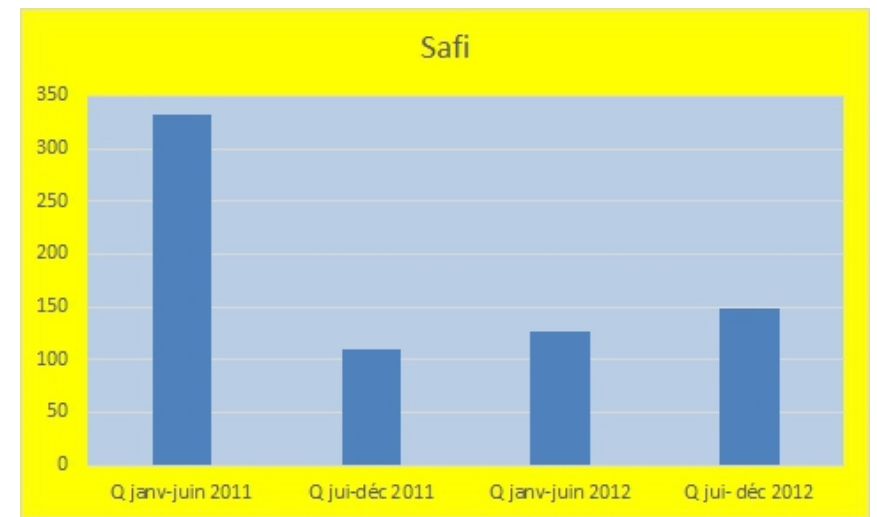

Figure 2 : Pluviométrie annuelle en mm de Safi en 2011 et 2012 (DMN) 

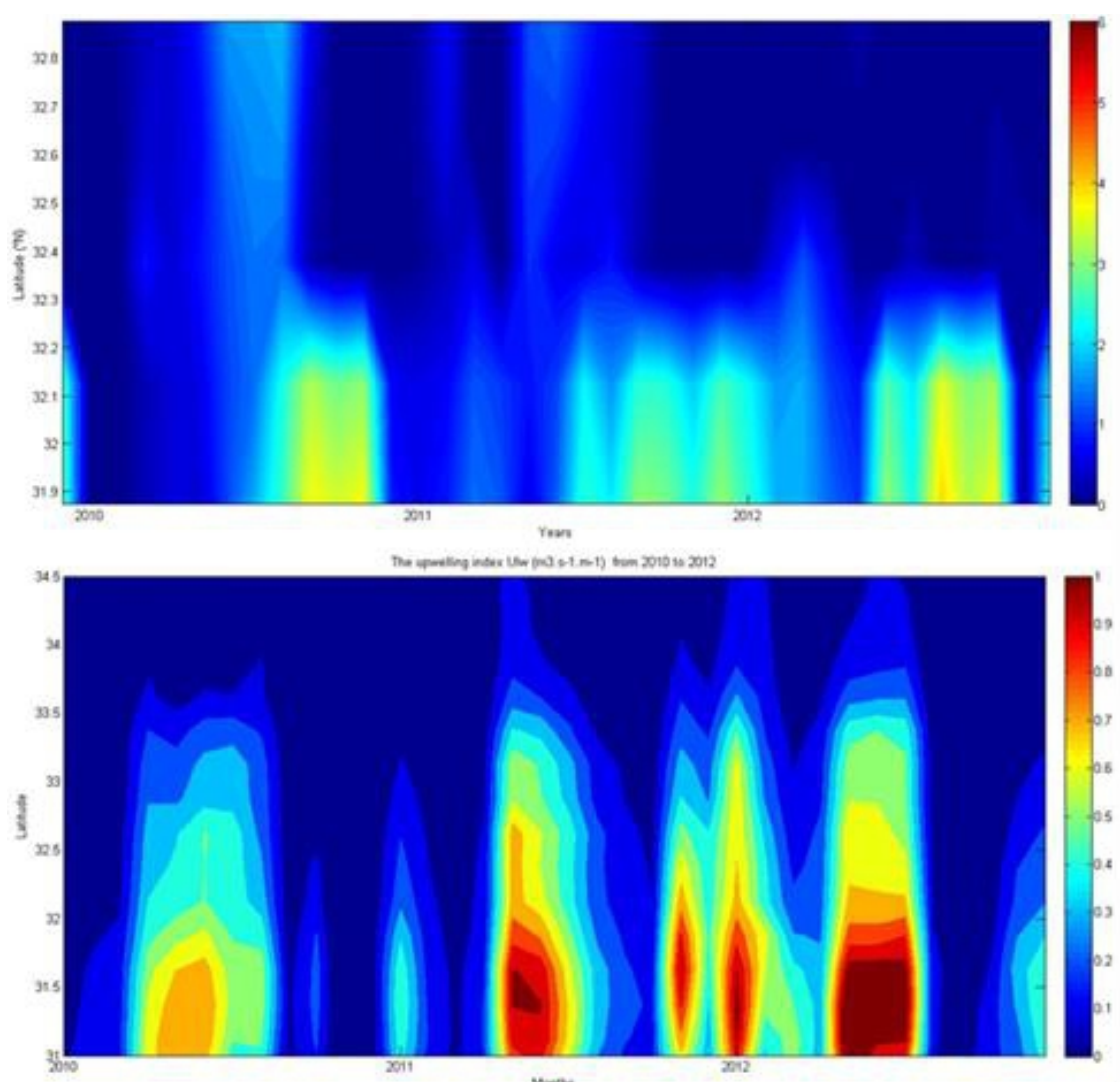

Figure 3 : Indice d'upwelling dérivé de la SST en haut et dérivé du vent en bas.

La fin de l'année 2011 et le début de l'année 2012 ont connu une faible abondance pluviométrique. Ce qui semble répercuter positivement sur les résurgences estivales de l'année 2012.

L'étude hydrologique de la lagune de Oualidia réalisée pendant l'année 2011 a mis en évidence une répartition hydrologique saisonnière et spatiale. En pleine mer, la lagune est plus influencée par l'envahissement des masses d'eau à caractéristique marine. Les eaux de la lagune à faible salinité de l'aval vers l'amont à basse mer, présentent à marée haute, des salinités dépassant 35 psu pratiquement au niveau de toute la lagune. Devant cette situation, les eaux de la lagune présentent une stabilité thermique durant les saisons du printemps et d'automne pendant lesquelles la température ne subit pas de forte variabilité diurnale aussi bien en amont qu'en aval. Par contre, pendant l'activité des résurgences d'eaux profondes en été, les eaux de la lagune subissent une forte variabilité thermique qui dépasse les $4^{\circ} \mathrm{C}$ au niveau de la partie centrale de la lagune où la température passe de $16^{\circ} \mathrm{C}$ à marée haute jusqu'à $20^{\circ} \mathrm{C}$ à marée basse. En hiver, les apports marins tendent à réchauffer la lagune en amont qui peut passer de $12.5^{\circ} \mathrm{C}$ à marée basse et atteindre $16^{\circ} \mathrm{C}$ à marée haute (Fig. 4). Cette structure se répète pratiquement 
pendant l'année 2012. La variabilité thermique saisonnière se manifeste par un refroidissement de la lagune durant toutes les saisons à l'exception de la saison d'hiver. Aussi, avons nous constaté une dessalure et un refroidissement de la lagune pendant l'année 2012 qui peut être dû à une activité plus importante des résurgences enregistrées durant la période estivale de cette année par rapport à l'année 2011 (Fig. 4). Les masses d'eau de la lagune présentées sur le diagramme TS (température-salinité) subissent une variabilité saisonnière plus marquée en été et en hiver et sont très influencées aussi bien par l'apport des eaux marines que par les résurgences d'eau douces continentales. La salinité de la lagune varie entre 20 et 35psu à marée basse, alors qu'à marée haute, elle atteint 30 à 36 psu pendant toutes les saisons. En hiver, les eaux océaniques tendent plus à réchauffer les eaux lagunaires. Par contre en été où les eaux océaniques sont froides par l'upwelling côtier atlantique, permettent un refroidissement de la lagune d'environ de $4^{\circ} \mathrm{C}$ de différence (Fig. 5).

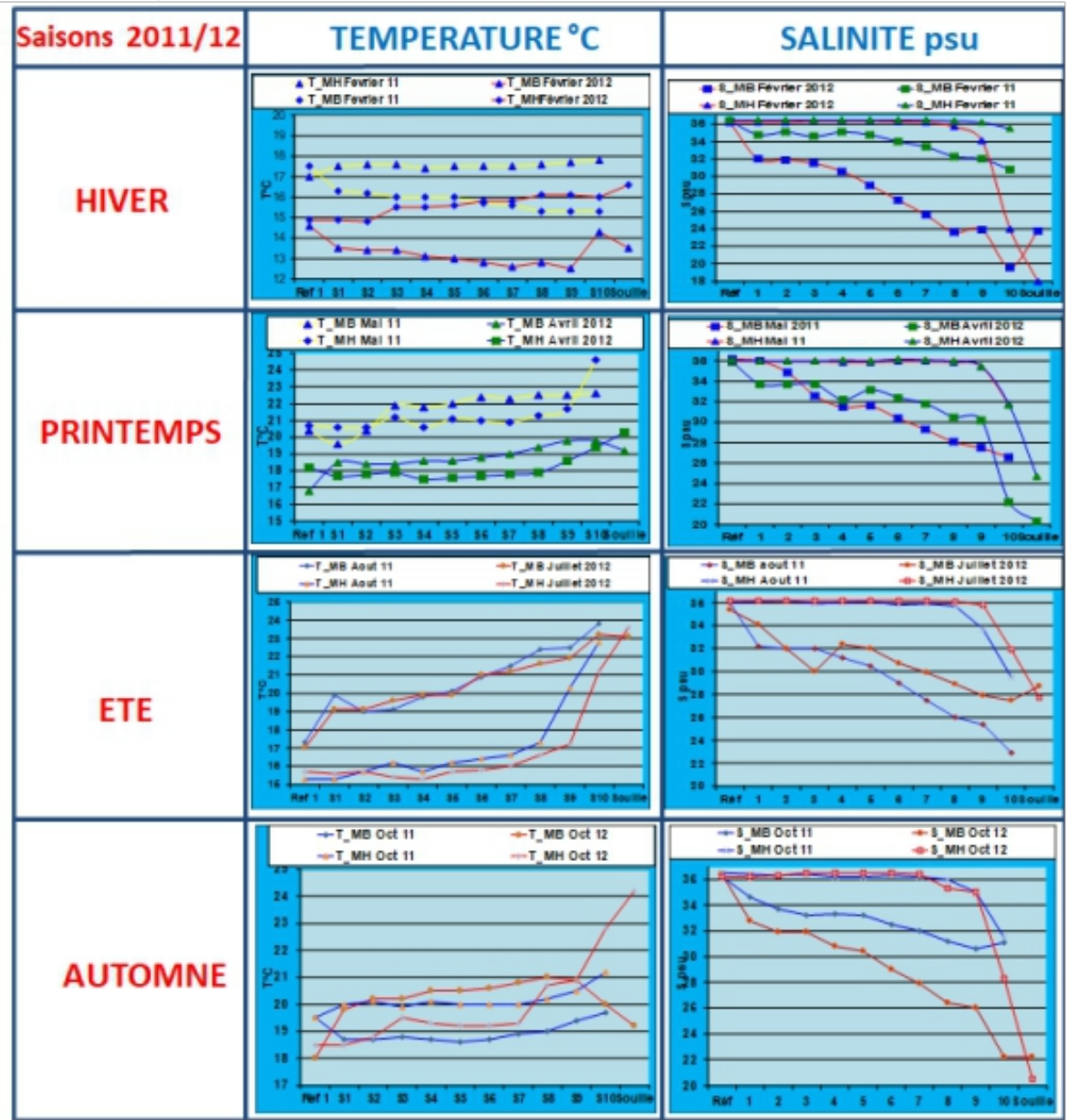

Figure 4 : Distribution de la température $(\mathrm{T})$ et de la salinité $(\mathrm{S})$ à marée basse $\left(\mathrm{T} \_\mathrm{MB}\right.$, S_MB) et marée haute (T_MH, S_MB) sur la période (2011 - 2012) 

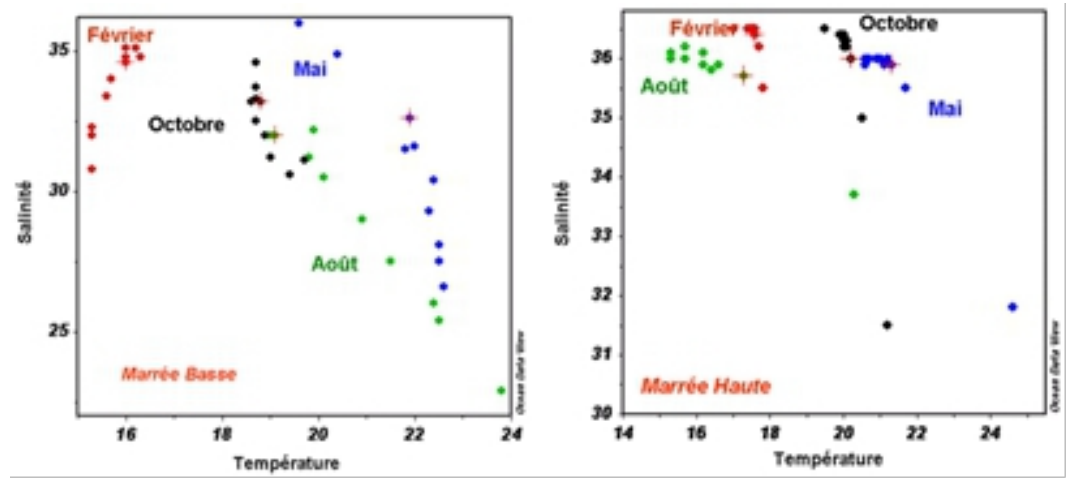

Figure 5 : Diagrammes température-salinité TS de la lagune de Oualidia pendant l'année 2011

Durant la période de construction de la souille en 2011, il a été observé une bonne oxygénation pendant toute l'année et encore plus à marée haute. Les eaux étaient aussi bien saturées en aval qu'en amont contrairement à l'étude réalisée par Damsiri et al. (2014) qui mentionnent une saturation en oxygène en amont plus importante qu'en aval pendant la saison du printemps. Durant l'année 2012, le même scénario se répète. Cependant, nous avons enregistré une diminution relative de la saturation de la lagune en oxygène dissous pendant cette année principalement pendant les saisons de l'été et l'automne à marée basse qui ont enregistré des concentrations moyennes de l'ordre de $5 \mathrm{ml} / \mathrm{l}$ (Fig. 6).

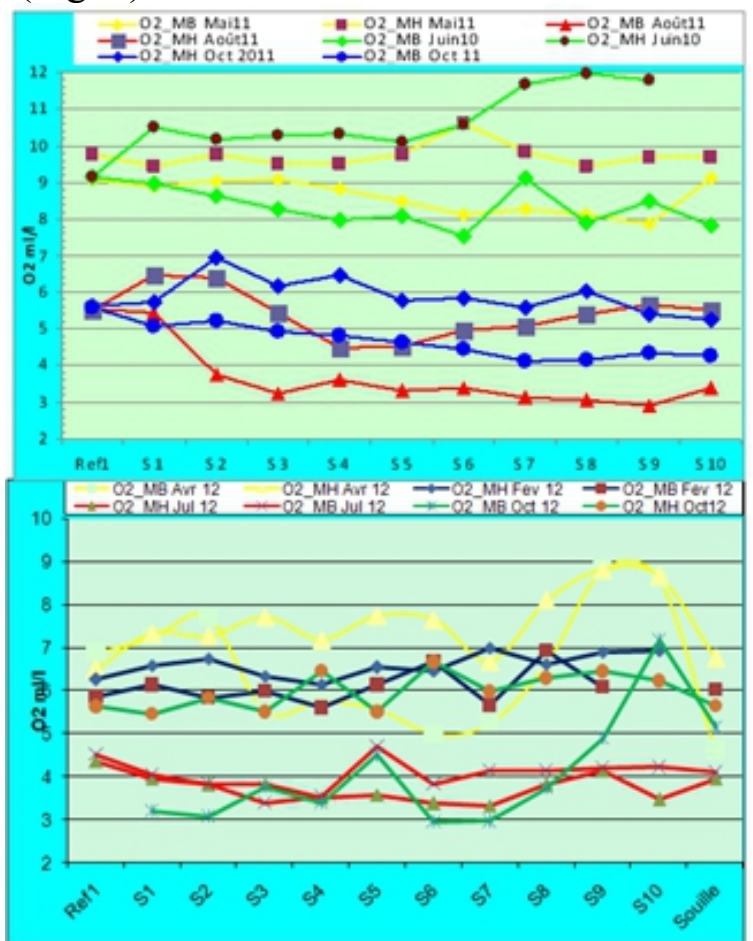

Figure 6 : Evolution des taux d'oxygène de la lagune de Oualidia (2011- 2012) 
La distribution des concentrations moyennes des nutriments de toutes les stations de la lagune présente une richesse importante notamment des nitrates et des ammoniums. Elles se manifestent par des concentrations plus élevées à marée basse qu'à marée haute. Les concentrations moyennes en ammoniums indiquent une augmentation notable pendant la saison du printemps principalement pendant l'année 2011 en atteignant $35 \mu \mathrm{M}$ et qui se sont réduites en 2012 en dépassant à peine $16 \mu \mathrm{M}$. Les résultats de Damsiri et al. (2014) indiquent une concentration plus importante en été $(+/-27 \mu \mathrm{M})$ qu'au printemps $(2.8 \mu \mathrm{M})$. Cette richesse en ammonium à marée basse montre son origine anthropique due principalement à l'agriculture, aux résurgences d'eau douce et aux rejets urbains. Quant aux concentrations moyennes des ortho phosphates, elles ont enregistré des taux élevés à marée haute et montrent un apport océanique important des phosphates qui ont connu une augmentation notable pendant l'année 2012. En effet, les concentrations ont atteint des valeurs très importantes à marée haute et sont passées de $2 \mu \mathrm{M}$ en 2011 à $7 \mu \mathrm{M}$ en 2012. Les concentrations moyennes des nitrates au niveau de la lagune ont été plus importantes à marée basse en dépassant $15 \mu \mathrm{M}$, mais sans enregistrer de variabilité entre les deux années à l'exception du mois d'août en 2011 et du mois de février 2012 qui ont connu des valeurs moyennes élevées dépassant des concentrations de $35 \mu \mathrm{M}$ à marée basse (Fig. 7).
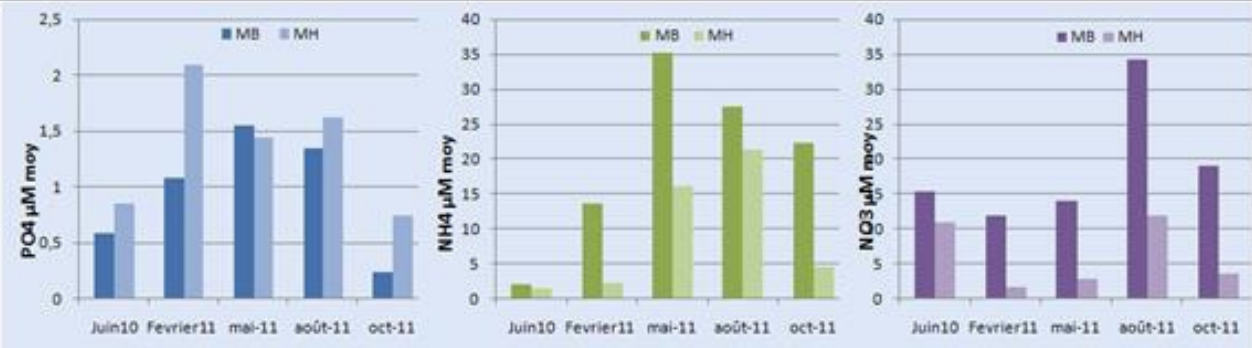

2011
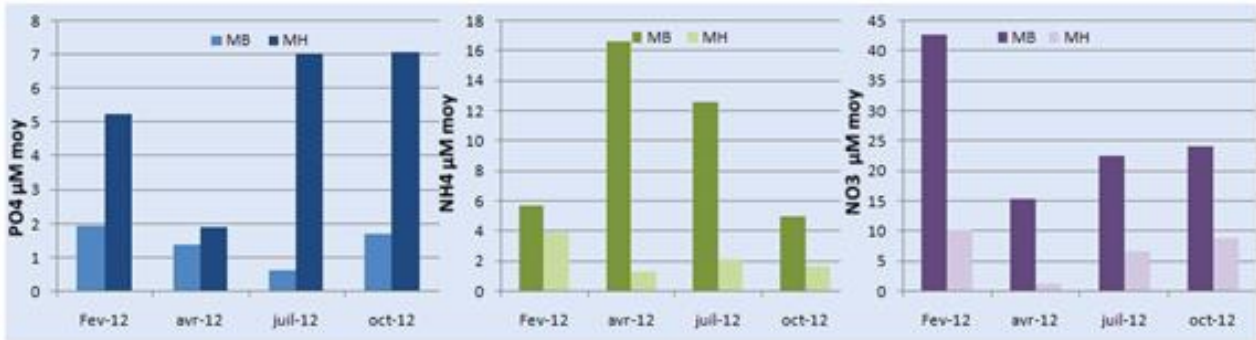

2012

Figure 7 : Distribution saisonnière de la teneur moyenne des nutriments (NO3, PO4 et NH4) en pleine mer (MH) et à basse mer (MB) (2011 - 2012) 
La richesse minérale à marée basse se reflète sur le développement important du phytoplancton qui se manifeste par une richesse importante en pigment chlorophyllien (Fig. 8). Il faut noter que cette richesse était plus importante pendant la construction de la souille en 2011 qui a causé la régénération des nutriments favorables à la productivité du milieu.

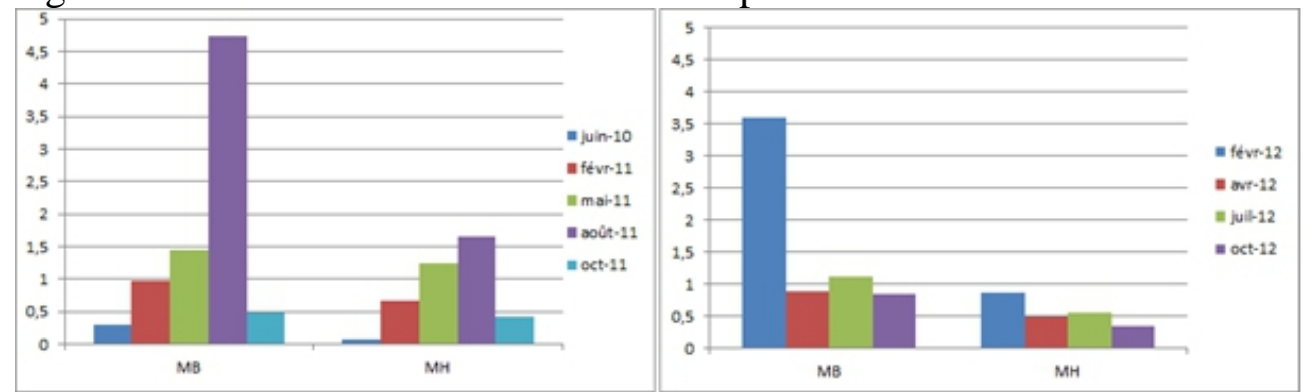

Figure 8 : Distribution saisonnière de la concentration moyenne de la Chlorophylle a $(\mu \mathrm{g} / \mathrm{l}))$ en pleine mer (MH) et à basse mer (MB) (2011 - 2012)

Les statistiques du courant (Fig. 9) montrent que le courant observé sur ce site a connu une amélioration remarquable après la construction de la souille.

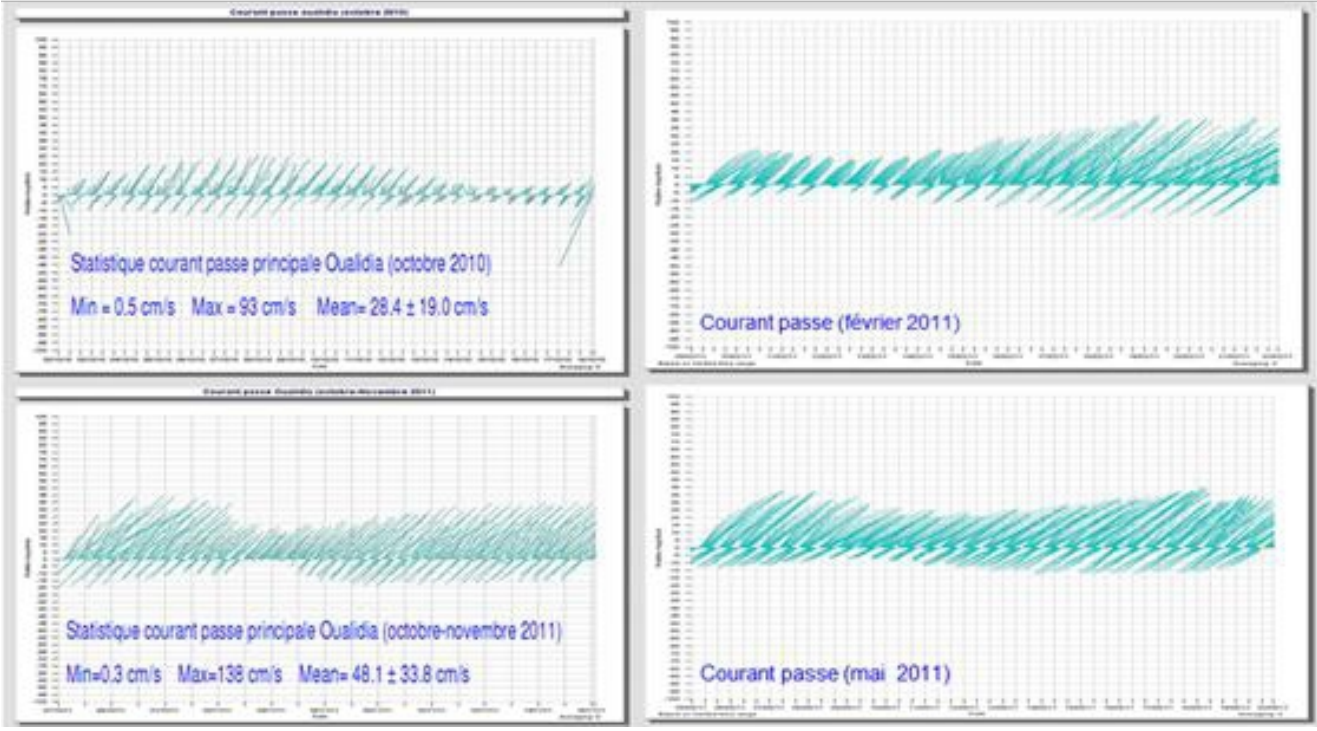

Figure 9 : Mesures du courant effectuées au niveau de la passe avant la construction de la souille (Octobre 2010) et après (Octobre 2011) au même point de mesures (Station S1)

Ainsi, durant la période d'étude actuelle, les intensités maximales sont de l'ordre de $138 \mathrm{~cm} / \mathrm{s}$ observées en octobre-novembre 2011, alors qu'elles ont été de l'ordre de $93 \mathrm{~cm} / \mathrm{s}$ en octobre 2010 (marées de vives-eaux avec coefficient de marée quasi comparables).

L'étude granulométrique des sables prélevés, en mois de février 2011 (période hivernale), le long du chenal de la lagune de Oualidia montre (Fig.10) 
la présence de deux faciès sédimentaires ; un faciès sableux et un faciès sable vaseux. Dans ces deux faciès, plus de $95 \%$ de ces éléments sont des arénites représentées généralement par des sables moyens. Par conséquent, le faciès sable vaseux est formé par un sédiment contenant $90 \%$ de sable et $10 \%$ constitue la vase dans des zones abritées du courant circulant dans le chenal principal. Ces études ont permis de mettre en évidence deux secteurs morpho - sédimentaires au niveau de la lagune de Oualidia. Un secteur en aval de la lagune au niveau de la passe caractérisé par un fort hydrodynamisme marin et un secteur en amont de la lagune caractérisé par un faible hydrodynamisme marin. Comme constaté par divers études précédentes (Hilmi et al., 2005a et b ; 2008 ; 2017), la répartition des faciès indique que l'hydrodynamisme de la lagune est décroissant de l'aval (Sable grossier) vers l'amont (vasière) et de la rive droite vers la rive gauche. Cette répartition coïncide parfaitement avec la richesse en matière organique en amont de la lagune par rapport à l'aval (Fig.

$10)$.

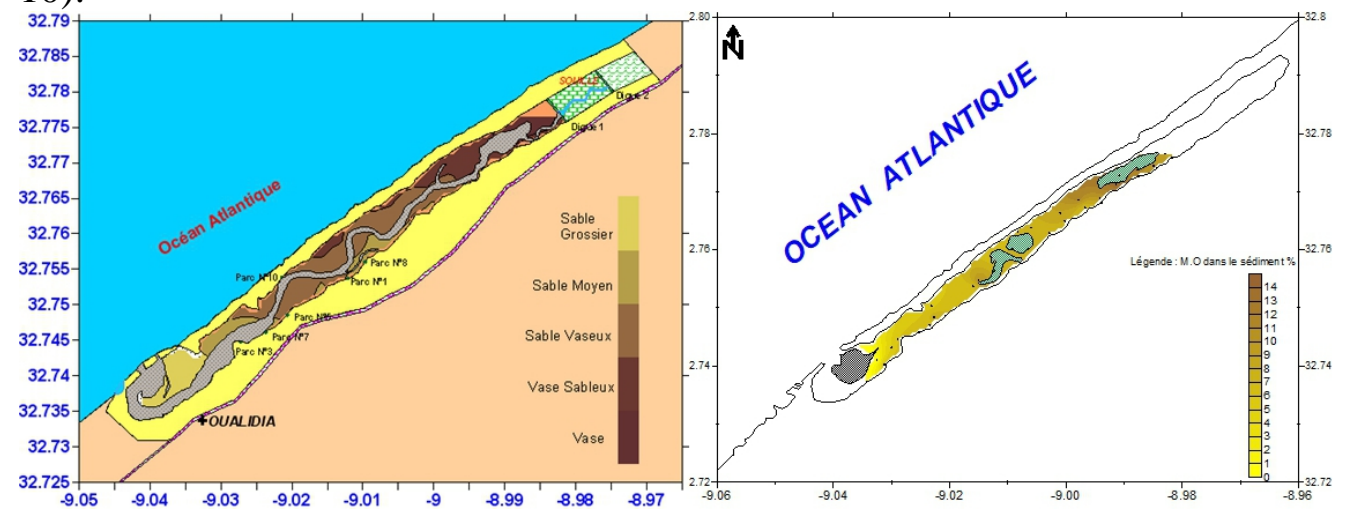

Figure 10 : Répartition des faciès (à gauche) et de la matière organique (à droite) dans la lagune de Oualidia (Août 2011)

Concernant les teneurs en matière organique mesurés au fond du chenal principal, elles fluctuent entre 1.16 et $9.10 \%$ (Fig. 11), et montrent un gradient croissant de l'aval vers l'amont avec des teneurs plus élevées au niveau des zones d'élevage des huîtres. En aval de la lagune, les teneurs sont faibles à cause de fort hydrodynamisme de cette zone qui ne permet pas la sédimentation de la fraction fine et par conséquent, la non sédimentation de la matière organique. Les études faites par Sarf (1999) ont montré un pourcentage élevé en matière organique entre 10.20 et $27.20 \%$. Cette diminution constaté est peut être due à la construction de la souille qui joue un rôle important dans le piégeage des sédiments fins et par conséquent le piégeage de la matière organique plus important vers l'amont comme il a été le cas pendant le mois d'août 2011. 


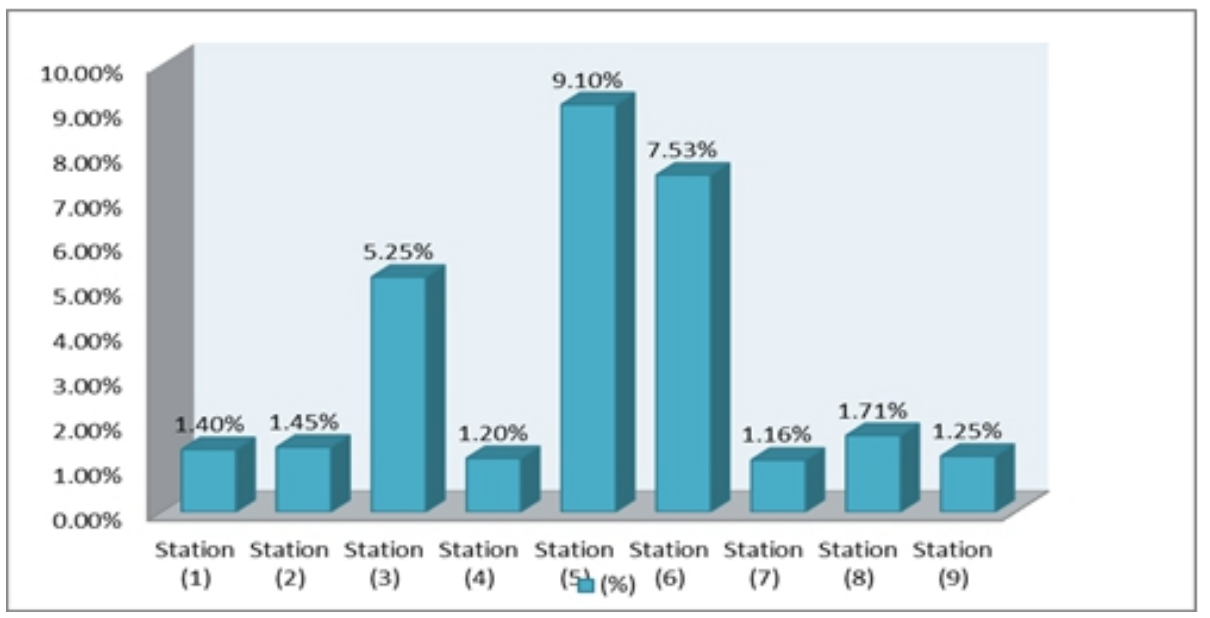

Figure 11 : Répartition de la matière organique le long du chenal principal de la lagune de Oualidia (février 2011)

\section{Conclusion}

Le suivi océanographique de l'écosystème marin de la lagune de oualidia réalisé sur la période de 2011-2012, nous a permis d'étudier l'état du milieu de la lagune de Oulaidia, notamment en 2011 où les travaux de la souille ont commencé.

Il a été constaté de manière générale, que la construction de la souille a favorisé un hydrodynamisme de la lagune accentué notamment au niveau de la sablière qui a changé de morphologie et qui s'est déplacée vers le Sud en créant une nouvelle structure du chenal principal. Cette hydrodynamique s'est répercutée en amont par une diminution de la matière organique sédimentaire qui est probablement due au piégeage des sédiments fins dans la souille.

\section{Remerciement}

Cet article est dédié à la mémoire de feu Dr Jaouad Lakhdar Idrissi, chercheur à l'Institut National de Recherche Halieutique qui a beaucoup travaillé de son vivant dans la lagune de Oualidia et la contribution de ces travaux à cette étude en reste témoins. Les auteurs tiennent aussi à remercier le personnel de la station RSSL de l'INRH à Oualidia pour leur appui aux missions de terrain ainsi que les évaluateurs externes de la revue.

\section{References:}

1. Beaubrun P.C, (1976). Les huîtres au Maroc et l'Ostréiculture dans la lagune de Oualidia. Bull. Inst. Pêches Marit. Maroc. N Spécial 176, 22, PP: 13-143.

2. Benbrahim S., Benhra A., Ennafah I, Khbaya B, Bouthir FZ, Bouhalaoui M., Taleb H, Koudad H, Cherkaoui R., Chfiri R., Jayed M., Nafil E., El Haimer B., Anhichem M., Bessa A., Moutaki B., 
Ettaouje D., Amnhir R., Benhadouche A., Frikech M., El Mourtaji H., Adil Q., Zemmouri H., A. Makaoui, A. Agouzouk, K. Hilmi, O. Ettahiri, Idhalla M., Belhcen O., Baibai T., Nhhala H., Zidane., M. Malouli, A., Marhoum et A. Faraj (2015). Lagune de Oualidia: Etat écologique et de santé environnementale, Institut national de recherche halieutique, Rapport INRH. Janvier 2015, 38p.

3. Bennouna A. (1999). Etude du phytoplancton nuisible et de son environnement dans la lagune de Oualidia et de Sidi Moussa (Maroc). Thèse de Doctorat 3ème Cycle, Faculté des Sciences, El Jadida, 153 p.

4. Benazzouz A., H. Demarcq, M. Chagdali, S. Mordane, A. Orbi, K. Hilmi, A. Atillah, J. Larissi, A. Makaoui, O. Ettahiri et A. Berraho (2014). Changement à long terme et tendance de l'activité de l'upwelling du systéme du courant des canaries à partir de l'imagerie satellite. Géo Observateur/ $\mathrm{N}^{\circ}$ 21, Mars 2014.

5. Bouhallaoui Mina, Ali Benhra, Bouchra El Haimeur, Françoise Quiniou et Mohammed Blaghen (2017). Utilisation du développement embryo larvaire de l'huitre creuse crassostrea gigas comme outil de diagnostic de la toxicité de substances pures et de mélanges complexes. Revue des Sciences de l'Eau 30(3) (2017) 171-181 ISSN : 1718-8598.

6. Carruesco C. (1989). Genèse et évolution de trois lagunes du littoral atlantique depuis l'halogène : Oualidia-Moulay Bou Selham (Maroc) et Arcachon (France). Thèse d'état $n^{\circ} 960$ tomme 1, Université de Bordeau I.

7. Chafik A., Cheggour M., Rharbi N. \& Bouhallaoui A. (1996). Evaluation de la salubrité de la lagune de Oualidia : étude de la contamination métallique de l'huître creuse Crassosteras gigas. Travaux et Documents, $\mathrm{N}^{\circ}$ 93, Institut National de Recherche Halieutique, Maroc.

8. Damsiri Zainab, Natij Laila, Khalil Karim, Loudiki Mohamed, Rabouille Christophe, Ettahiri Omar, Bougadir Belaid, Elkalay Khalid. (2014). Spatio-temporal nutrients variability in the Oualidia lagoon (Atlantic Moroccan coast) International Journal of Advanced Research (2014), Volume 2, Issue 8, 609-618.

9. El Attar J. (1998). Contribution à la détermination de l'origine de la contamination fécale dans la lagune de Oualidia (Maroc) et étude de la contamination bactériologique de l'huître "Crassostera Gigas 》 en conditions naturelles et expérimentales. Thèse de 3ème Cycle, Faculté des Sciences, El Jadida, 124 p.

10. Hilmi K. (2005). Modélisation numérique de la circulation de deux milieux paraliques du littoral marocain : la lagune de Oualidia 
(Atlantique) et la lagune de Nador (Méditerranée), Thèse Univ. Hassan II-Mohammedia, Fac. Sci. Ben Msik, Casablanca, 186 p.

11. Hilmi Karim, Abdellatif Orbi, Jaouad I. Lakhdar et Farida Sarf (2005a). Etude courantologique de la lagune de Oualidia (Maroc) en automne. Bulletin de 1'Institut Scientifique, Rabat, section Sciences de la Vie, 2004-2005, n²6-27, 67-71.

12. Hilmi K., Koutitonsky V.G., Orbi A., Lakhdar J.I. \& Chagdali M. (2005b). Oualidia lagoon: an estuary without a river. Afr. J. Aquatic Sci., 30, 1, 1-10.

13. Hilmi Karim, Makaoui Ahmed, Ettahiri Omar, Idrissi Mohammed, Larissi Jamila, Abdellaoui Benyounes, El Ouehabi Zineb and et Orbi Abdellatif (2017). Fonctionnement hydrodynamique de la lagune de Oualidia (Maroc) avant l'aménagement de la souille. ISSN: 2320-5407 Int. J. Adv. Res. 5(7), 2015-2027.

14. Hilmi K. A., Orbi A. \& J. Lakhdar Idrissi (2008). Modélisation de la circulation de la lagune de Oualidia durant l'été et l'automne 2005. Travaux et Documents INRH, 116, 54 p.

15. Koutitonsky, V.G., Orbi, A., Ouabi, M. et Ibrahimi I. (2006). L'étude du comportement hydro-sédimentaire du système lagunaire Oualidia par la modélisation mathématique. Phase 1 : Synthèse des données et simulations de la réfraction des houles. Direction des Ports et du Domaine Public Maritime, Ministère de l'Équipement et du Transport, Royaume du Maroc. $150 \mathrm{p}$.

16. Lakhdar Idrissi J., Orbi A., Hilmi K., Zidane F., Sarf F., Massik Z .et A. Makaoui (2004). Organisation et fonctionnement d'un écosystème côtier du maroc: la lagune de Khnifiss. Revue Sciences de l'Eau, 17(4), 447-462.

17. Mejjad N., A. Laissaoui, O. El-Hammoumi, M. Benmansour, S., Benbrahim, H. Bounouira, A., Benkdad, F. Z. Bouthir, A. Fekri, M. Bounakhla (2016). Sediment geochronology and geochemical behavior of major and rare earth elements in the Oualidia Lagoon in the western Morocco. J Radioanal Nucl Chem DOI 10.1007/s10967016-4714-8.

18. Makaoui A., A. Orbi, K. Hilmi, S. Zizah, J. Larissi and M. Talbi (2005). L'upwelling de la côte atlantique du Maroc entre 1994 et 1998. C. R. Geoscience 337 (2005) 1518-1524.

19. Makaoui A., Orbi, A., Arestigui, J., Azzouz, A. B., Laarissi, J., Agouzouk, A. and Hilmi, K., (2012). Hydrological seasonality of cape Ghir filament in Morocco. Natural Science, Vol.4, No.1, 5-13.

20. Makaoui Ahmed, Aziz Agouzouk, Tarik Baibai, Mohammed Idrissi, Jamila Larissi, Ismail Bessa, Soukaina Obad, Omar Ettahiri, Mustapha Ait Chattou et Karim Hilmi (2017). Caractéristiques océanographiques 
de la zone littorale de Dakhla - Oued Eddahab et son impact sur l'hydrodynamique marine de la baie de Cintra (Maroc). International Journal of Advanced Research (IJAR), Volume 5, Issue 7, 2055-2068 p.

21. Orbi A., Hilmi K., Larissi J., Zidane H., Zizah S., El Moussaoui N., Lakhdar J.I. \& Sarf F. (1998). Hydrologie et hydrodynamique des côtes marocaines : milieux paraliques et zones côtières. Commissariat général Expo'98, Lisbonne, 68 p.

22. Rharbi N. (2000). Importance des paramètres hydrologiques et phytoplanctoniques sur la croissance de l'huître Crassostrea gigas en élevage dans la lagune de Oualidia. Thèse d•Etat, Faculté des Sciences Ben Msik, Casablanca, 217 p.

23. Rharbi N., Ramdani M., Berraho Ab. \& Lakhdar J.I. (2001). Caractéristiques hydrologiques et écologiques de la lagune de Oualidia : milieu paralique de la côte atlantique marocaine. Marine Life, 11, 12, 3-9.

24. Sarf F. (1999). Dynamique sédimentaire et état de pollution de la lagune de Oualidia. Thèse de 3ème Cycle, Faculté des Sciences, Rabat, $121 \mathrm{p}$. 


\section{Annexe}

\begin{tabular}{|c|c|c|c|c|c|c|c|c|}
\hline \multirow{2}{*}{ Station } & \multicolumn{2}{|c|}{ juin-10 } & \multicolumn{2}{c|}{ mai-11 } & \multicolumn{2}{c|}{ août-11 } & \multicolumn{2}{c|}{ oct-11 } \\
\cline { 2 - 10 } & MB & MH & MB & MH & MB & MH & MH & MB \\
\hline Ref1 & 9,13 & 9,13 & 9,07 & 9,76 & 5,56 & 5,50 & 5,61 & 5,57 \\
\hline S1 & 8,95 & 10,50 & 8,90 & 9,42 & 5,45 & 6,48 & 5,75 & 5,08 \\
\hline S2 & 8,62 & 10,17 & 9,03 & 9,76 & 3,74 & 6,38 & 6,96 & 5,24 \\
\hline S3 & 8,25 & 10,28 & 9,07 & 9,52 & 3,23 & 5,43 & 6,17 & 4,95 \\
\hline S4 & 7,97 & 10,30 & 8,80 & 9,52 & 3,61 & 4,47 & 6,48 & 4,81 \\
\hline S5 & 8,08 & 10,10 & 8,50 & 9,76 & 3,31 & 4,52 & 5,77 & 4,64 \\
\hline S6 & 7,54 & 10,57 & 8,13 & 10,60 & 3,39 & 4,98 & 5,84 & 4,46 \\
\hline S7 & 9,12 & 11,66 & 8,27 & 9,82 & 3,13 & 5,09 & 5,60 & 4,12 \\
\hline S8 & 7,90 & 11,95 & 8,12 & 9,42 & 3,06 & 5,39 & 6,01 & 4,17 \\
\hline S9 & 8,47 & 11,78 & 7,86 & 9,68 & 2,92 & 5,68 & 5,41 & 4,36 \\
\hline S10 & 7,82 & - & 9,09 & 9,71 & 3,40 & 5,51 & 5,27 & 4,27 \\
\hline Souille & - & - & - & - & - & - & - & - \\
\hline
\end{tabular}

Tableau 1 : Concentration moyenne d'oxygène dissous $(\mathrm{m} / \mathrm{l})$ dans la lagune de Oualidia (2011) en pleine mer (MH) et à basse mer (MB)

\begin{tabular}{|c|c|c|c|c|c|c|c|c|}
\hline \multirow{2}{*}{ Station } & \multicolumn{2}{|c|}{ févr-12 } & \multicolumn{2}{c|}{ avr-12 } & \multicolumn{2}{c|}{ juil-12 } & \multicolumn{2}{c|}{ oct-12 } \\
\cline { 2 - 9 } & MH & MB & MB & MH & MH & MB & MB & MH \\
\hline Ref1 & 6,26 & 5,86 & 6,90 & 6,51 & 4,36 & 4,52 & - & 5,64 \\
\hline S1 & 6,60 & 6,13 & 7,24 & 7,34 & 3,96 & 4,04 & 3,21 & 5,46 \\
\hline S2 & 6,74 & 5,83 & 7,72 & 7,27 & 3,81 & 3,83 & 3,07 & 5,84 \\
\hline S3 & 6,35 & 6,00 & 5,52 & 7,72 & 3,82 & 3,39 & 3,76 & 5,51 \\
\hline S4 & 6,15 & 5,61 & 5,75 & 7,20 & 3,51 & 3,53 & 3,40 & 6,47 \\
\hline S5 & 6,54 & 6,14 & 5,56 & 7,75 & 3,58 & 4,69 & 4,50 & 5,51 \\
\hline S6 & 6,46 & 6,69 & 5,00 & 7,65 & 3,38 & 3,82 & 2,96 & 6,67 \\
\hline S7 & 6,99 & 5,67 & 5,24 & 6,69 & 3,34 & 4,15 & 2,96 & 5,98 \\
\hline S8 & 6,62 & 6,93 & 6,64 & 8,12 & 3,81 & 4,15 & 3,73 & 6,29 \\
\hline S9 & 6,91 & 6,09 & 8,81 & 8,83 & 4,16 & 4,20 & 4,89 & 6,45 \\
\hline S10 & 6,94 & - & 8,61 & 8,68 & 3,47 & 4,22 & 7,17 & 6,24 \\
\hline Souille & - & 6,02 & 4,63 & 6,76 & 3,96 & 4,10 & 5,16 & 5,66 \\
\hline
\end{tabular}

Tableau 2 : Concentration moyenne d'oxygène dissous $(\mathrm{m} / \mathrm{l})$ dans la lagune de Oualidia (2012) en pleine mer (MH) et à basse mer (MB) 


\begin{tabular}{|c|c|c|c|c|c|c|}
\hline \multirow{2}{*}{ Période } & \multicolumn{2}{|c|}{ NH4 } & \multicolumn{2}{c|}{ PO4 } & \multicolumn{2}{c|}{ NO3 } \\
\cline { 2 - 7 } & MB & MH & MB & MH & MB & MH \\
\hline juin-10 & 2,11 & 1,57 & 0,58 & 0,86 & 15,45 & 11,05 \\
\hline févr-11 & 13,72 & 2,36 & 1,08 & 2,09 & 11,88 & 1,80 \\
\hline mai-11 & 35,16 & 16,23 & 1,55 & 1,44 & 14,00 & 2,95 \\
\hline août-11 & 27,53 & 21,43 & 1,35 & 1,62 & 34,17 & 11,86 \\
\hline oct-11 & 22,36 & 4,64 & 0,24 & 0,74 & 19,06 & 3,69 \\
\hline févr-12 & 5,76 & 3,91 & 1,95 & 5,26 & 42,69 & 10,34 \\
\hline avr-12 & 16,67 & 1,34 & 1,38 & 1,93 & 15,46 & 1,32 \\
\hline juil-12 & 12,66 & 2,13 & 0,65 & 7,06 & 22,51 & 6,65 \\
\hline oct-12 & 5,00 & 1,70 & 1,71 & 7,08 & 24,27 & 8,92 \\
\hline
\end{tabular}

Tableau 3 : Concentration moyenne des nutriments (NO3, PO4 et NH4 $(\mu \mathrm{mol} / \mathrm{l})$ ) en pleine mer (MH) et à basse mer (MB) (2011 - 2012)

\begin{tabular}{|l|l|l|}
\hline \multirow{2}{*}{ Période } & \multicolumn{2}{l|}{ Concentration de Chlorophylle « $\mathbf{a}$ » en } \\
\cline { 2 - 4 } & MB & MH \\
\hline juin-10 & 0,30 & 0,06 \\
\hline févr-11 & 0,97 & 0,66 \\
\hline mai-11 & 1,46 & 1,23 \\
\hline août-11 & 4,73 & 1,66 \\
\hline oct-11 & 0,48 & 0,41 \\
\hline févr-12 & 3,59 & 0,86 \\
\hline avr-12 & 0,89 & 0,49 \\
\hline juil-12 & 1,11 & 0,56 \\
\hline oct-12 & 0,85 & 0,34 \\
\hline
\end{tabular}

Tableau 4 : Concentration moyenne de la Chlorophylle a $(\mu \mathrm{g} / \mathrm{l})$ ) en pleine mer $(\mathrm{MH})$ et à basse mer (MB) (2011 - 2012), 